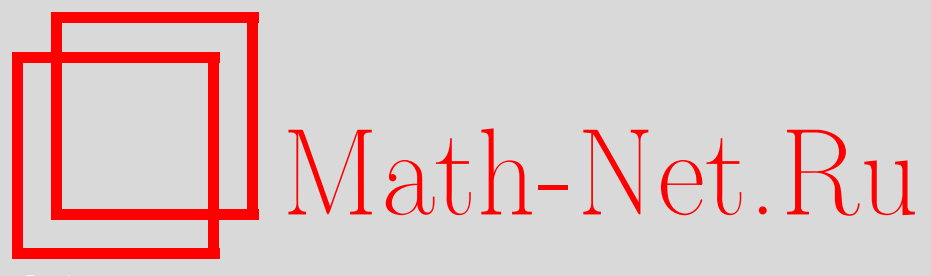

И. В. Павлов, О. В. Назарько, Обобщение теоремы Дуба о свободном выборе для деформированных субмартингалов, УМН, 2013, том 68, выпуск 6, 175-176

DOI: https://doi.org/10.4213/rm9560

Использование Общероссийского математического портала Math-Net.Ru подразумевает, что вы прочитали и согласны с пользовательским соглашением http://www . mathnet.ru/rus/agreement

Параметры загрузки:

IP : 52.90 .164 .192

26 апреля 2023 г., 11:41:06

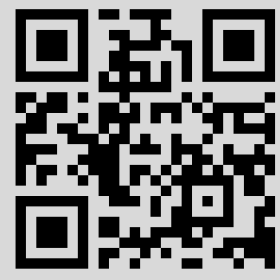




\title{
Обобщение теоремы Дуба о свободном выборе для деформированных субмартингалов
}

\author{
И. В. Павлов, О. В. Назарько
}

Данная работа посвящена развитию теории преобразования свободного выбора в случае дискретного времени (см., например, [1]) на структуре, значительно более общей, чем классический стохастический базис. Эта структура названа авторами деформированным стохастическим базисом и представляет собой фильтрованное пространство вместе с некоторым семейством вероятностей.

Пусть $(\Omega, \mathbf{F})$ - фильтрованное пространство с дискретным временем, где $\Omega$ - произвольное множество, а $\mathbf{F}=\left(\mathscr{F}_{n}\right)_{n=0}^{\infty}-$ возрастающая последовательность $\sigma$-алгебр на нем (фильтрация). Семейство $\mathbf{Q}=\left(\mathbf{Q}^{(n)}, \mathscr{F}_{n}\right)_{n=0}^{\infty}$ вероятностных мер $\mathbf{Q}^{(n)}$, определенных на $\mathscr{F}_{n}$, будем называть деформацией (выбор такого термина подробно аргументирован в [2]). Назовем $\mathbf{Q}$ деформацией 1-го рода (D1), если при всех $n \in \mathbb{N}=$ $\{0,1,2, \ldots\}$ выполняются соотношения абсолютной непрерывности $\left.\mathbf{Q}^{(n+1)}\right|_{\mathscr{F}_{n}} \ll \mathrm{Q}^{(n)}$, и деформацией 2-го рода (D2), если выполняются соотношения $\left.\mathrm{Q}^{(n+1)}\right|_{\mathscr{F}_{n}} \gg \mathrm{Q}^{(n)}$.

Предположим, что при всех $n \in \mathbb{N}$ случайные величины (с. в.) $Z_{n}$ принадлежат пространствам $L_{p}\left(\Omega, \mathscr{F}_{n}, \mathbf{Q}^{(n)}\right)$. Если $\mathbf{Q}$ есть D1 (соответственно D2), то процесс $\mathbf{Z}=$ $\left(Z_{n}, \mathscr{F}_{n}, \mathrm{Q}^{(n)}\right)_{n=0}^{\infty}$ назовем деформированным субмартингалом 1-го рода - DSubM1 (соответственно 2-го рода - DSubM2) при выполнении $\mathrm{Q}^{(n+1)}$-п. н. (соответственно $\mathrm{Q}^{(n)}$-п. н.) неравенств $Z_{n} \leqslant \mathrm{E}^{\mathrm{Q}^{(n+1)}}\left[Z_{n+1} \mid \mathscr{F}_{n}\right]$ для всех $n \in \mathbb{N}$. Аналогично определяются деформированные супермартингалы и мартингалы 1-го и 2-го рода (DSupM1, DSupM2, DM1, DM2).

Пусть $\tau: \Omega \rightarrow \mathbb{N}$ - конечный момент остановки (м. о.) относительно фильтрации $\left(\mathscr{F}_{n}\right)_{n=0}^{\infty}$ (в данной работе мы будем рассматривать только такие м. о.). Пусть также $\mathbf{Q}$ - произвольная деформация. Для любого $A \in \mathscr{F}_{\tau}$ обозначим $\mathbf{Q}^{(\tau)}(A)=$ $\sum_{i=0}^{\infty} \mathrm{Q}^{(i)}(A\{\tau=i\})$. Ясно, что $\mathrm{Q}^{(\tau)}$ - неотрицательная $\sigma$-конечная мера, совпадающая на $\{\tau=n\}$ при любом $n \in \mathbb{N}$ с мерой $\mathrm{Q}^{(n)}$. Если м. о. $\tau$ принимает лишь конечное число значений, то мера $Q^{(\tau)}$ ограничена. В дальнейшем, если интеграл по мере $\mathrm{Q}^{(\tau)}$ от $\mathscr{F}_{\tau}$-измеримой с. в. $f$ определен, то он обозначается $\mathrm{E}^{(\tau)}(f)=\mathrm{E}^{\mathrm{Q}^{(\tau)}}(f)$.

Следующие технические результаты, использующиеся в доказательствах основных теорем данной работы, представляют и самостоятельный интерес.

ПрЕДЛОЖЕНИЕ 1. Пусть м.о. $\tau$ и $\nu$ таковы, что $0 \leqslant \nu-\tau \leqslant 1$, c. в. $f$ измерима относительно $\mathscr{F}_{\nu}$ и либо $f \geqslant 0 \mathrm{Q}^{(\nu)}$-n.в., либо $f \in L_{1}\left(\Omega, \mathscr{F}_{\nu}, \mathrm{Q}^{(\nu)}\right)$. Тогда $\mathrm{Q}^{(\nu)}$-n.в. справедливо равенство

$$
\mathrm{E}^{(\nu)}\left[f \mid \mathscr{F}_{\tau}\right]=\sum_{k=0}^{\infty} f I_{\{\tau=k\}} I_{\{\nu=k\}}+\sum_{k=0}^{\infty} \mathrm{E}^{(k+1)}\left[f I_{\{\nu=k+1\}} \mid \mathscr{F}_{k}\right] I_{\{\tau=k\}} I_{\{\nu=k+1\}} .
$$

ПреДЛОЖениЕ 2. Пусть м.о. $\tau$ и $\nu$ таковы, ито $0 \leqslant \nu-\tau \leqslant N<\infty$. Если $\mathbf{Q}$ есть D1, mо $\left.\mathrm{Q}^{(\nu)}\right|_{\mathscr{F}_{\tau}} \ll \mathrm{Q}^{(\tau)}$. Eсли $\mathbf{Q}$ есть D2, mо $\left.\mathbf{Q}^{(\tau)} \ll \mathrm{Q}^{(\nu)}\right|_{\mathscr{F}_{\tau}}$.

ПредЛОЖенИЕ 3. Пусть м. о. $\tau$ ограничен, а $\mathbf{Q}$ естъ D1 или D2. Тогда $\mathbf{Q}^{(\tau)}(\Omega)>0$.

Отметим, что если м. о. $\tau$ неограничен, то легко конструируются примеры деформаций 1-го и 2-го рода, для которых $\mathrm{Q}^{(\tau)}(\Omega)=0$.

$\mathrm{C}$ помощью предложений 1 и 2 доказывается следующая теорема, неулучшаемая для D1 и обобщаемая в дальнейшем для D2.

Работа выполнена при поддержке РФФИ (грант № 13-01-00637-а).

DOI: $10.4213 / \mathrm{rm} 9560$ 
TeOpema 1. Пусть $\left(Z_{n}, \mathscr{F}_{n}, \mathrm{Q}^{(n)}\right)_{n=0}^{\infty}$ ecmъ DSubM, м. о. $\tau$ и $\nu$ таковы, что $0 \leqslant$ $\nu-\tau \leqslant 1$ u $Z_{\nu} \in L_{1}\left(\Omega, \mathscr{F}_{\nu}, \mathrm{Q}^{(\nu)}\right)$. Если $\mathbf{Q}$ есть D1, mo $\mathrm{E}^{(\nu)}\left[Z_{\nu} \mid \mathscr{F}_{\tau}\right] \geqslant\left. Z_{\tau} \mathrm{Q}^{(\nu)}\right|_{\mathscr{F}_{\tau}}{ }^{-n . в .}$ Если $\mathbf{Q}$ есть D2, то $\mathrm{E}^{(\nu)}\left[Z_{\nu} \mid \mathscr{F}_{\tau}\right] \geqslant Z_{\tau} \mathbf{Q}^{(\tau)}-n .8$.

Для деформации 2-го рода $\mathbf{Q}$ введем процесс плотностей $\left(h^{(n)}, \mathscr{F}_{n}\right)_{n=0}^{\infty}$ формулой $d \mathbf{Q}^{(n)}=\left.h^{(n)} d \mathbf{Q}^{(n+1)}\right|_{\mathscr{F}_{n}}$. Будем говорить, что деформация $\mathbf{Q}$ ограничена (BD2), если $\left\|h^{(n)}\right\|_{L_{\infty}\left(\Omega, \mathscr{F}_{n}, \mathrm{Q}^{(n)}\right)}<\infty$ для любого $n \in \mathbb{N}$. Равномерная ограниченность (UBD2) определяется неравенством $\sup _{n}\left\|h^{(n)}\right\|_{L_{\infty}\left(\Omega, \mathscr{F}_{n}, \mathrm{Q}^{(n)}\right)}<\infty$.

Пусть $0 \leqslant \nu-\tau \leqslant 1$. Рассмотрим операторы $E_{\tau}^{(\nu)} f:=\mathrm{E}^{(\nu)}\left[f \mid \mathscr{F}_{\tau}\right]$. Нетрудно доказать, что если $\mathbf{Q}$ есть D2, то $E_{\tau}^{(\nu)}: L_{\infty}\left(\Omega, \mathscr{F}_{\nu}, \mathrm{Q}^{(\nu)}\right) \rightarrow L_{\infty}\left(\Omega, \mathscr{F}_{\tau}, \mathrm{Q}^{(\tau)}\right)$ - ограниченный линейный оператор с нормой 1 , и если $\mathbf{Q}$ есть UBD2, то $E_{\tau}^{(\nu)}: L_{p}\left(\Omega, \mathscr{F}_{\nu}, \mathrm{Q}^{(\nu)}\right) \rightarrow$ $L_{p}\left(\Omega, \mathscr{F}_{\tau}, \mathrm{Q}^{(\tau)}\right)$ - ограниченный линейный оператор для любого $1 \leqslant p<\infty$.

ОПРеДЕлЕНИЕ 1 . Пусть $\mathbf{Q}$ есть D2 и $\tau \leqslant \nu$ - два м. о., удовлетворяющие условию $0 \leqslant \nu-\tau \leqslant N<\infty$. Образуем следующую последовательность м. о. $\left\{\tau_{k}\right\}_{k=0}^{K}$ :

$$
\tau=\tau_{0} \leqslant \tau_{1} \leqslant \cdots \leqslant \tau_{K}=\nu, \quad 0 \leqslant \tau_{k+1}-\tau_{k} \leqslant 1, \quad k=0,1, \ldots, K-1
$$

(например, можно положить $\left.\tau_{k}=\nu \wedge(\tau+k), k=0,1, \ldots, N\right)$. Если $f-\mathscr{F}_{\nu}$-измеримая с. в. такая, что $f \geqslant 0 \mathrm{Q}^{(\nu)}$-п. в., то определим оператор $E_{\tau}^{(\nu)} f=E_{\tau_{0}}^{\left(\tau_{1}\right)} E_{\tau_{1}}^{\left(\tau_{2}\right)} \cdots E_{\tau_{K-1}}^{\left(\tau_{K}\right)} f$.

Теорема 2. В условиях определения $1 \mathrm{Q}^{(\tau)}$-п.в. справедлива формула (обосновывающая корректность определения $E_{\tau}^{(\nu)}: E_{\tau}^{(\nu)} f=\sum_{k=0}^{\infty} I_{\{\tau=k\}} \sum_{i=0}^{\infty} E_{k}^{(k+i)}\left(f I_{\{\nu=k+i\}}\right)$.

ТЕорема 3 (обобщение теоремы Дуба о преобразовании свободного выбора). Предположим, что $\mathbf{Q}$ есть UBD2, $\mathbf{Z}=\left(Z_{n}, \mathscr{F}_{n}, \mathrm{Q}^{(n)}\right)_{n=0}^{\infty}$ есть DSubM, $0 \leqslant \nu-\tau \leqslant N<\infty$ и $Z_{\nu} \in L_{1}\left(\Omega, \mathscr{F}_{\nu}, \mathrm{Q}^{(\nu)}\right)$. Справедливо неравенство

$$
E_{\tau}^{(\nu)} Z_{\nu} \geqslant Z_{\tau} \quad \mathrm{Q}^{(\tau)}-n .8 .
$$

Теоремы 1 и 3 с очевидными видоизменениями справедливы для деформированных супермартингалов и мартингалов.

СледСтвиЕ 1. Теорема о преобразовании свободного выбора справедлива, если $\mathbf{Q}$ есть BD2 в случае, когда м.о. $\nu$ принимает конечное число значений.

ЗАмечание 1 . Пусть $\mathrm{P}$ - вероятностная мера, определенная на $\mathscr{F}_{\infty}$ - наименьшей $\sigma$-алгебре, содержащей все $\mathscr{F}_{n}(n \in \mathbb{N})$, а $\mathrm{Q}^{(n)}=\left.\mathrm{P}\right|_{\mathscr{F}_{n}}$. Тогда следствие 1 совпадает с классической теоремой о преобразовании свободного выбора для ограниченных м. о. Что касается основной теоремы 3 , то для классического стохастического базиса $\left(\Omega, \mathbf{F}, \mathscr{F}_{\infty}, \mathrm{P}\right)$ при условиях $0 \leqslant \nu-\tau \leqslant N<\infty$ и $Z_{\nu} \in L_{1}\left(\Omega, \mathscr{F}_{\nu}, \mathrm{P}\right)$ получаем выполнение Р-п. н. неравенства $\mathrm{E}\left(Z_{\nu} \mid \mathscr{F}_{\tau}\right) \geqslant Z_{\tau}$. Возможно, такое достаточное условие является новым.

\section{Список литературы}

[1] А.Н. Ширяев, О мартингалъных методах в задачах о пересечении грании, броуновским движением, Совр. пробл. матем., 8, МИАН, М., 2007, 80 с. [2] О. В. Назарько, И. В. Павлов, Вестн. Ростов. гос. ун-та путей сообщения, 2012, № 1(45), 200-208.

И. В. Павлов (I. V. Pavlov)

Ростовский государственный строительный университет E-mail: pavloviv2005@mail.ru

O. В. Назарько (O. V. Nazarko)

Ростовский государственный строительный университет

E-mail: pavloviv2005@mail.ru
Представлено Д. В. Трещёвым Принято редколлегией 05.10 .2013 